\title{
Providing a Heating Degree Days (HDDs) Atlas across Iran Entire Zones
}

\author{
M. Mehrabi ${ }^{1, *}$, A. Kaabi-Nejadian ${ }^{1,2}$, M. Khalaji Asadi ${ }^{1}$ \\ ${ }^{1}$ Department of Environment and Energy, Science and Research Branch, Islamic Azad University, Tehran, Iran \\ ${ }^{2}$ Renewable Energy Organization of Iran, Ministry of Energy, Tehran, Iran \\ *Corresponding author. Tel: +98912 6431993, Fax: +9821 44696541,E-mail: meh_mehrabi@yahoo.com
}

\begin{abstract}
Considering fossil fuels depletion and increasing of energy demand in Iran, a special attention is required toward the energy conservation. Energy demand of building section in Iran is very high, which is as a result of many factors such as governmental huge subsidies for energy, lack of energy conservation culture in building inhabitants, poor insulation of buildings and poor heating or cooling control systems.

Most of buildings heating control systems in Iran do not respond properly to weather temperature changes during winters, therefore most of the time the interior temperature of these buildings exceed the comfort temperature, thus these buildings are not energy efficient and consume excessive amount of energy. The most important index to identify these buildings across the country is to know HDDs for each point of the country.

Unfortunately, up to now no comprehensive research has been conducted in Iran about HDDs, and thus no HDDs atlas has been provided, therefore it is essential for energy managers, engineers and in particular for the government to be supplied with HDDs for each point of Iran. By taking this fact into account, we decided to prepare a comprehensive HDDs atlas for Iran entire zones.

In this paper authorized temperature databases of 255 meteorological stations in 30 provinces of Iran have been collected from Iran meteorological organization, thereafter HDDs for each station were calculated, then a mathematical modeling (multiple regression analysis technique) was employed in order to simulate the HDDs of other places in Iran. Consequently, a HDDs Atlas across Iran entire zones was provided.

These results can widely be used in energy consumption planning and prediction of the heating energy demand in buildings and enhances the government abilities to manage the rate of energy consumption in buildings.
\end{abstract}

Keywords: Iran heating HDDs atlas, Energy management.

\section{Nomenclature}

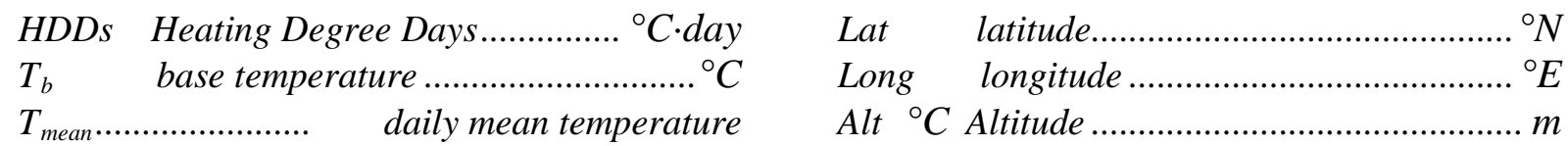

\section{Introduction}

Unfortunately, until now no comprehensive research about HDDs has been conducted in Iran. In this paper authorized daily temperature databases of 255 meteorological stations in 30 provinces of Iran have been collected, thereafter the annually HDDs for each station were calculated. Then a mathematical modeling (multiple regression analysis technique) was employed to simulate the HDDs of other places. Consequently, a HDDs Atlas across Iran was provided.

\section{Methodology}

Fundamentally HDDs are a summation of the differences between the outdoor temperature and base temperature over a specified time period. HDDs are a useful tool that can be used in the assessment of weather related energy consumption in buildings, according to Eq. (1).

$$
\begin{aligned}
& \text { Heating energy demand }(\mathrm{kWh})= \\
& \qquad \begin{array}{l}
\text { Overall heat loss coefficient }\left(\mathrm{kW} \cdot{ }^{\circ} \mathrm{C}^{-1}\right) \times \operatorname{HDDs}\left({ }^{\circ} \mathrm{C} \cdot \text { day }\right) \times 24\left(\mathrm{~h} \cdot \mathrm{day}^{-1}\right) \\
(\text { The } 24 \text { is included to convert from days to hours. })
\end{array}
\end{aligned}
$$

In current study accessible authorized daily temperature databases have been collected from 255 meteorological stations (Fig. 1) during last 5 years. 


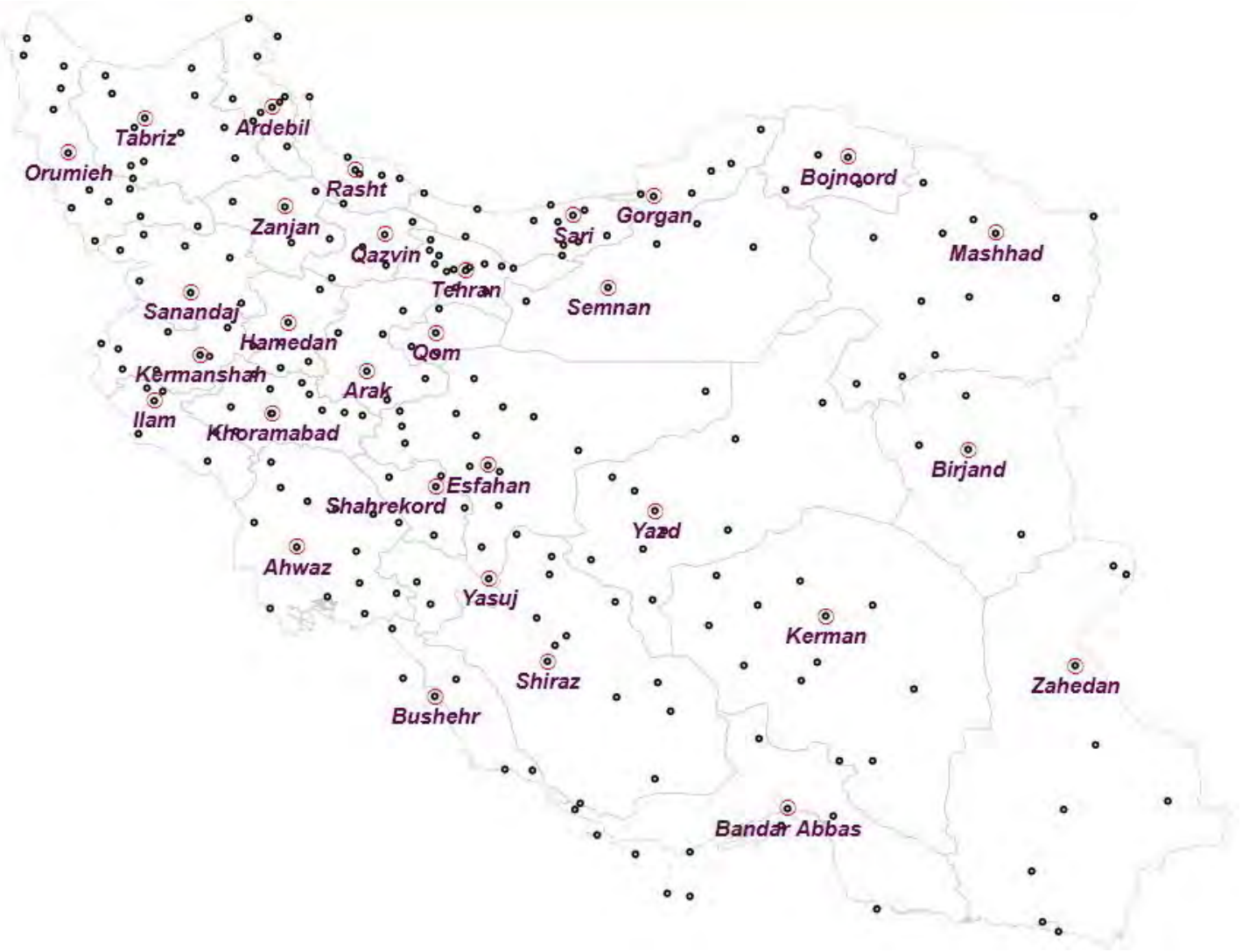

Fig. 1. Location of 255 Iran official meteorological stations in 30 provinces.

In this paper mean daily temperature method has been employed that is generally used in countries, such as USA [1] and Germany [2], where HDDs are calculated from the mean daily temperature. This makes the definition and calculation of HDDs simpler, and makes the reasonable assumption that efficient heating systems do not operate on days where outdoor temperature averages exceed the base temperature [3]. In this method we have applied Eq. (2).

$\left\{\begin{array}{lll}\text { HDDs }=T_{b}-T_{\text {mean }} & \text { if } & T_{b}>T_{\text {mean }} \\ \text { HDDs }=0 & \text { if } & T_{b} \leq T_{\text {mean }}\end{array}\right.$

(Based on local conditions, we assume $\mathrm{T}_{\mathrm{b}}=18^{\circ} \mathrm{C}$ )

To calculate HDDs, at first the mean temperature was calculated for each day of the year, Thereafter, by applying Eq. (2) HDDs were calculated. Then by summation of HDDs during each year, we obtained annually HDDs during 5 years, subsequently annually HDDs average during this period attained (Table.1.).

Afterwards by computerizing the calculated HDDS, spline multiple regression analysis technique was employed in order to simulate the annual HDDs over each other point of country. In this method, a two dimensional function (surface) has been constructed closely fits range of a discrete set of known data points (HDDS in $255 \mathrm{~s}$ tations), so we could estimate HDDs values in other points of country. Spline surfaces are very popular in computerized regression because of the simplicity of their construction, their ease and accuracy of evaluation, and their capacity to approximate complex shapes through surface fitting and interactive design [4]. In this paper by constructing spline surface from calculated HDDs, the comprehensive HDDs Atlases were provided (Fig.2. and Fig.3.). 


\section{Results}

In Table 1, for each 255 meteorological stations longitude, latitude, elevation and calculated annual HDDs average have been determined. These results show various kinds of climate zones in Iran.

Table 1. Calculated annual HDDs in Iran meteorological stations.

\begin{tabular}{|c|c|c|c|c|c|c|c|c|c|}
\hline Station Name & Lat & Long & Alt & $\overline{\text { HDDs }}$ & Station Name & Lat & Long & Alt & HDD \\
\hline Abadan & 30.37 & 48.25 & 7 & 428 & Boinzahra & 35.77 & 50.07 & 1282 & 2098 \\
\hline Abadeh & 31.18 & 52.67 & 2030 & 2076 & Bojnoord & 37.47 & 57.32 & 1091 & 2319 \\
\hline Abali & 35.75 & 51.88 & 2465 & 3544 & Bonab & 37.33 & 46.07 & 1290 & 2549 \\
\hline Abarkuh & 31.13 & 53.28 & 1524 & 1387 & Bookan & 36.53 & 46.22 & 1386 & 2600 \\
\hline Abbor & 36.93 & 48.97 & 703 & 1503 & Borazjan & 29.25 & 51.17 & 90 & 263 \\
\hline Abumusa island & 25.83 & 54.83 & 7 & 5 & Borujen & 31.95 & 51.30 & 2197 & 2937 \\
\hline Aghda & 32.43 & 53.62 & 1150 & 1173 & Borujerd & 33.92 & 48.75 & 1629 & 2184 \\
\hline Ahar & 38.43 & 47.07 & 1391 & 2869 & Bostan & 31.72 & 48.00 & 8 & 550 \\
\hline Ahwaz & 31.33 & 48.67 & 23 & 406 & Bostan Abad & 37.85 & 46.85 & 1750 & 3533 \\
\hline Alasht & 36.08 & 52.85 & 190 & 2820 & Bushehr & 28.98 & 50.83 & 20 & 253 \\
\hline Aleshtar & 33.82 & 48.25 & 1567 & 2476 & Chahbahar & 25.28 & 60.62 & 8 & 12 \\
\hline Aliabad & 36.90 & 54.87 & 140 & 1414 & Chalderan & 39.07 & 44.38 & 1788 & 3811 \\
\hline Aligudarz & 33.40 & 49.70 & 2022 & 2649 & Chitgar & 35.70 & 51.13 & 1215 & 1737 \\
\hline Amol & 36.47 & 52.38 & 24 & 1333 & Damavand & 35.72 & 52.07 & 1960 & 2928 \\
\hline Anar & 30.88 & 55.25 & 1409 & 1337 & Damqan & 36.10 & 54.32 & 1155 & 1801 \\
\hline Anzali & 37.47 & 49.47 & -26 & 1408 & Darab & 28.75 & 54.53 & 1140 & 777 \\
\hline Aqdasieh & 35.78 & 51.62 & 1548 & 1911 & Daran & 32.97 & 50.37 & 2290 & 3042 \\
\hline Arak & 34.10 & 49.77 & 1708 & 2363 & Darehshahr & 33.13 & 47.40 & 670 & 1065 \\
\hline Ardebill & 38.33 & 48.40 & 1314 & 3414 & Dashtenaz & 36.63 & 53.18 & 20 & 1318 \\
\hline Ardebil2 & 38.25 & 48.28 & 1332 & 3049 & Dayyer & 27.83 & 51.93 & 4 & 100 \\
\hline Ardestan & 33.38 & 52.38 & 1252 & 1466 & Dehdasht & 30.78 & 50.55 & 820 & 889 \\
\hline Astara & 38.42 & 48.87 & -18 & 1676 & Dehdoz & 31.72 & 50.27 & 1457 & 1448 \\
\hline Avaj & 35.57 & 49.22 & 2035 & 3087 & Dehloran & 32.68 & 47.27 & 232 & 473 \\
\hline Azna & 33.45 & 49.42 & 1872 & 2801 & Delijan & 33.98 & 50.68 & 1524 & 1964 \\
\hline Babolsar & 36.72 & 52.65 & -21 & 1196 & Deylams & 30.05 & 50.17 & 4 & 359 \\
\hline Badrabad & 33.43 & 48.27 & 1155 & 1619 & Dezful & 32.27 & & 83 & 523 \\
\hline Bafq & 31.60 & 55.43 & 991 & 950 & Dogonbadan & 30.43 & 50.77 & 700 & 711 \\
\hline Baft & 29.23 & 56.58 & 2280 & 1837 & Dorud & 33.48 & 49.07 & 1527 & 1953 \\
\hline Bam & 29.10 & 58.35 & 1067 & 628 & Doshantappeh & 35.70 & 51.33 & 1209 & 1433 \\
\hline Bandar Abbas & 27.22 & 56.37 & 10 & 86 & Eivane Qarb & 33.83 & 46.32 & 1170 & 1675 \\
\hline Bandar Torkaman & 36.88 & 54.07 & -20 & 1314 & Eqlid & 30.90 & 52.63 & 2300 & 2371 \\
\hline Baneh & 36.00 & 45.90 & 1600 & 2459 & Esfahan & 32.62 & 51.67 & 1550 & 1952 \\
\hline Bavanat & 30.47 & 53.67 & 2231 & 2148 & Esfarayen & 37.05 & 57.48 & 1216 & 2172 \\
\hline Behbahan & 30.60 & 50.23 & 313 & 537 & Eslam abad & 34.12 & 46.47 & 1349 & 2276 \\
\hline Beshruyeh & 33.90 & 57.45 & 885 & 1450 & Fasa & 28.97 & 53.68 & 1288 & 1142 \\
\hline Biarjmand & 36.05 & 55.83 & 1106 & 1905 & Ferdos & 34.02 & 58.17 & 1293 & 1587 \\
\hline Bijar & 35.88 & 47.62 & 1883 & 3014 & Firuzkuh & 35.92 & 52.83 & 1976 & 3479 \\
\hline Bileh Savar & 39.37 & 48.37 & 90 & 1937 & Gariz & 31.30 & 54.10 & 2100 & 2194 \\
\hline Birjand & 32.87 & 59.20 & 1491 & 1651 & Garmsar & 35.20 & 52.27 & 825 & 1444 \\
\hline
\end{tabular}


Table 1 (continued). Calculated annual HDDs in Iran meteorological stations.

\begin{tabular}{|c|c|c|c|c|c|c|c|c|c|}
\hline Station & Lat & Long & Alt & HDDs & Station & Lat & Long & Alt & HDDs \\
\hline Geophysics & 35.73 & 51.38 & 1419 & 1725 & Kish island & 26.50 & 53.98 & 30 & 17 \\
\hline Germi & 39.05 & 48.05 & 749 & 2222 & Komijan & 34.70 & 49.32 & 1741 & 2734 \\
\hline Gilaneqarb & 34.13 & 45.93 & 816 & 1167 & Kuhdasht & 33.53 & 47.63 & 1200 & 1818 \\
\hline Golmakan & 36.48 & 59.28 & 1176 & 2259 & Kuhrang & 32.43 & 50.12 & 2285 & 3398 \\
\hline Golpayegan & 33.47 & 50.28 & 1870 & 2318 & Kushk Nosrat & 35.08 & 50.90 & 948 & 1292 \\
\hline Gonabad & 34.35 & 58.68 & 1056 & 1646 & Lahijan & 37.18 & 50.00 & 86 & 1445 \\
\hline Gonbade Kavous & 37.25 & 55.17 & 37 & 1252 & Lalehzar & 29.52 & 56.83 & 2775 & 3127 \\
\hline Gorgan & 36.85 & 54.27 & 13 & 1338 & Lamerd & 27.30 & 53.12 & 411 & 353 \\
\hline Haji Abad & 28.32 & 55.92 & 931 & 638 & Lar & 27.68 & 54.28 & 792 & 561 \\
\hline Hamedan & 34.87 & 48.53 & 1742 & 2805 & Lavan & 26.80 & 53.38 & 22 & 26 \\
\hline Hashtgerd & 36.00 & 50.75 & 1613 & 2314 & Lengeh & 26.53 & 54.83 & 23 & 40 \\
\hline Hendijan & 30.28 & 49.73 & 3 & 440 & Lordegan & 31.52 & 50.82 & 1580 & 1865 \\
\hline Hoseinieh & 32.67 & 48.27 & 354 & 494 & Mahabad & 36.77 & 45.72 & 1385 & 2435 \\
\hline Ilam & 33.63 & 46.43 & 1337 & 1776 & Mahneshan & 36.77 & 47.67 & 1282 & 2305 \\
\hline Imam Airport & 35.42 & 51.17 & 990 & 1675 & Mahshahr & 30.55 & 49.15 & 6 & 409 \\
\hline Iranshahr & 27.20 & 60.70 & 591 & 248 & Makoo & 39.33 & 44.43 & 1411 & 3126 \\
\hline Izadkhast & 31.53 & 52.12 & 2188 & 2299 & Malayer & 34.25 & 48.85 & 1778 & 2459 \\
\hline Izeh & 31.85 & 49.87 & 767 & 913 & Malekan & 37.13 & 46.10 & 1300 & 2501 \\
\hline Jajerm & 36.95 & 56.33 & 984 & 1966 & Maneh & 37.50 & 56.85 & 890 & 1962 \\
\hline Jam-Tohid & 27.82 & 52.37 & 655 & 534 & Manjil & 36.73 & 49.40 & 333 & 1343 \\
\hline Jask & 25.63 & 57.77 & 5 & 10 & Marand & 38.47 & 45.77 & 1550 & 2891 \\
\hline Jolfa & 38.75 & 45.67 & 736 & 2363 & Maraqeh & 37.40 & 46.27 & 1478 & 2445 \\
\hline Kabutar Abad & 32.52 & 51.85 & 1545 & 1885 & Maravetapp & 37.90 & 55.95 & 460 & 1409 \\
\hline Kahak & 34.40 & 50.87 & 1403 & 1884 & Marivan & 35.52 & 46.20 & 1287 & 2463 \\
\hline Kahnooj & 27.97 & 57.70 & 470 & 304 & Marvast & 30.50 & 54.25 & 1547 & 1415 \\
\hline Kalaleh & 37.37 & 55.48 & 150 & 1324 & Mashhad & 36.27 & 59.63 & 999 & 1904 \\
\hline Kaleibar & 38.87 & 47.02 & 1180 & 2540 & Masjed & 31.93 & 49.28 & 321 & 535 \\
\hline Kangavar & 34.50 & 47.98 & 1468 & 2518 & Mehran & 33.12 & 46.18 & 150 & 711 \\
\hline Karaj & 35.92 & 50.90 & 1313 & 2003 & Mehriz & 31.58 & 54.43 & 1520 & 1319 \\
\hline Kashan & 33.98 & 51.45 & 982 & 1498 & Meshkin & 38.38 & 47.67 & 1569 & 2951 \\
\hline Kashmar & 35.20 & 58.47 & 1110 & 1588 & Meybod & 32.22 & 53.97 & 1108 & 1411 \\
\hline Kenarak & 25.43 & 60.37 & 12 & 41 & Meymeh & 33.43 & 51.17 & 1980 & 2742 \\
\hline Kerman & 30.25 & 56.97 & 1754 & 1538 & Miandoab & 36.97 & 46.05 & 1300 & 2586 \\
\hline Kermanshah & 34.35 & 47.15 & 1319 & 2012 & Mianeh & 37.45 & 47.70 & 1110 & 2359 \\
\hline Khalkhal & 37.63 & 48.52 & 1796 & 3566 & Minab & 27.10 & 57.08 & 30 & 62 \\
\hline Khansar & 33.23 & 50.32 & 2300 & 2752 & Moallemkelay & 36.45 & 50.48 & 1629 & 2325 \\
\hline Khark & 29.27 & 50.33 & 4 & 205 & Murche Khort & 33.08 & 51.48 & 1669 & 1927 \\
\hline Khash & 28.22 & 61.20 & 1394 & 914 & Nahavand & 34.15 & 48.42 & 1681 & 2398 \\
\hline Khodabandeh & 36.12 & 48.58 & 1887 & 3037 & Nahbandan & 31.53 & 60.03 & 1211 & 1179 \\
\hline Khomein & 33.65 & 50.08 & 1835 & 2346 & Najafabad & 32.60 & 51.38 & 1641 & 1798 \\
\hline Khor Birjand & 32.93 & 58.43 & 1117 & 1316 & Namin & 38.42 & 48.48 & 1450 & 3055 \\
\hline Khoramabad & 33.43 & 48.28 & 1148 & 1625 & Naqdeh & 36.95 & 45.42 & 1338 & 2598 \\
\hline Khoramdarreh & 36.18 & 49.18 & 1575 & 2584 & Natanz & 33.53 & 51.90 & 1685 & 1993 \\
\hline Khorbiabanak & 33.78 & 55.08 & 845 & 1186 & Nayin & 32.85 & 53.08 & 1549 & 1692 \\
\hline Khoy & 38.55 & 44.97 & 1103 & 2660 & Nayyer & 38.03 & 47.98 & 1600 & 2932 \\
\hline Kiasar & 36.23 & 53.53 & 1294 & 2263 & Neyriz & 29.20 & 54.33 & 1632 & 1149 \\
\hline
\end{tabular}


Table 1 (continued). Calculated annual HDDs in Iran meteorological stations.

\begin{tabular}{|c|c|c|c|c|c|c|c|c|c|}
\hline Station Name & Lat & Long & Alt & $\overline{\text { HDDs }}$ & Station Name & Lat & Long & Alt & HDDs \\
\hline Neyshabur & 36.27 & 58.80 & 1213 & 2129 & Saravan & 27.33 & 62.33 & 1195 & 655 \\
\hline Nikshahr & 26.23 & 60.20 & 510 & 91 & Sardasht & 36.15 & 45.50 & 1670 & 2429 \\
\hline Noshahr & 36.65 & 51.50 & -21 & 1415 & Sare Ein & 38.17 & 48.10 & 1632 & 3303 \\
\hline Nourabad & 34.05 & 48.00 & 1859 & 2798 & Sari & 36.55 & 53.00 & 23 & 1225 \\
\hline Omidieh & 30.77 & 49.65 & 35 & 437 & Sarpolezahab & 34.45 & 45.87 & 545 & 1075 \\
\hline Orumieh & 37.53 & 45.08 & 1316 & 2694 & Saveh & 35.05 & 50.33 & 1108 & 1600 \\
\hline Parsabad & 39.65 & 47.92 & 32 & 1922 & Semirom & 31.33 & 51.57 & 2274 & 2568 \\
\hline Parsian & 27.20 & 53.03 & 70 & 84 & Semnan & 35.42 & 53.55 & 1131 & 1610 \\
\hline Payam Karaj & 35.78 & 50.83 & 1261 & 2153 & Shahdad & 30.42 & 57.70 & 400 & 447 \\
\hline Piranshahr & 36.67 & 45.13 & 1455 & 2513 & Shahrebabak & 30.10 & 55.13 & 1834 & 1870 \\
\hline Poldokhtar & 33.15 & 47.72 & 714 & 899 & Shahrekord & 32.28 & 50.85 & 2049 & 3020 \\
\hline Polesefid & 36.13 & 53.08 & 610 & 1703 & Shahreza & 31.98 & 51.83 & 1845 & 2067 \\
\hline Qaen & 33.72 & 59.17 & 1432 & 2001 & Shahriar & 35.67 & 51.02 & 2986 & 1817 \\
\hline Qarakhil & 36.45 & 52.77 & 15 & 1348 & Shahrud & 36.42 & 54.95 & 1345 & 1956 \\
\hline Qare Ziaeddin & 38.90 & 45.02 & 1108 & 2724 & Shiraz & 29.53 & 52.60 & 1484 & 1331 \\
\hline Qasre Shirin & 34.53 & 45.60 & 376 & 860 & Shushtar & 32.05 & 48.83 & 67 & 390 \\
\hline Qazvin & 36.25 & 50.05 & 1279 & 2168 & Siahbisheh & 36.22 & 51.32 & 2165 & 2881 \\
\hline Qeshm island & 26.95 & 56.27 & 13 & 64 & Silakhor & 33.73 & 48.87 & 1497 & 2186 \\
\hline Qom & 34.70 & 50.85 & 877 & 1553 & Siri island & 25.88 & 54.48 & 4 & 12 \\
\hline Qorveh & 35.17 & 47.80 & 1906 & 2887 & Sirjan & 29.47 & 55.68 & 1739 & 1419 \\
\hline Quchan & 37.07 & 58.50 & 1287 & 2509 & Sonqor & 34.78 & 47.58 & 1700 & 2568 \\
\hline Rafsanjan & 30.42 & 55.90 & 1581 & 1270 & Tabas & 33.60 & 56.92 & 711 & 929 \\
\hline Ramhormoz & 31.27 & 49.60 & 151 & 406 & Tabriz & 38.08 & 46.28 & 1361 & 2555 \\
\hline Ramsar & 36.90 & 50.67 & -20 & 1376 & Tafresh & 34.68 & 50.02 & 1979 & 2582 \\
\hline Rasht1 & 37.27 & 49.58 & -10 & 1507 & Takab & 36.38 & 47.12 & 1765 & 3353 \\
\hline Rasht2 & 37.20 & 49.65 & 37 & 1457 & Takestan & 36.05 & 49.70 & 1283 & 2199 \\
\hline Ravansar & 34.72 & 46.65 & 1380 & 2159 & Takhtjams & 29.93 & 52.90 & 1605 & 1487 \\
\hline Razan & 35.38 & 49.03 & 1840 & 2902 & Taleqan & 36.17 & 50.77 & 1857 & 2920 \\
\hline Robat & 33.03 & 55.55 & 1188 & 1378 & Tehran & 35.68 & 51.32 & 1191 & 1495 \\
\hline Rudan & 27.97 & 57.18 & 220 & 84 & Torbate Hey. & 35.27 & 59.22 & 1451 & 2230 \\
\hline Rudsar & 37.13 & 50.28 & -19 & 1463 & Torbate Jam & 35.25 & 60.58 & 950 & 1969 \\
\hline Sabzevar & 36.20 & 57.72 & 978 & 1669 & Tuyserkan & 34.55 & 48.43 & 1783 & 2471 \\
\hline Sad Dorudzan & 30.22 & 52.43 & 1620 & 1482 & Varamin & 35.35 & 51.63 & 927 & 1603 \\
\hline Sahand & 37.93 & 46.12 & 1641 & 2783 & Yasuj & 30.83 & 51.68 & 1832 & 1927 \\
\hline Salafchegan & 34.48 & 50.47 & 1381 & 1799 & Yazd & 31.90 & 54.28 & 1237 & 1185 \\
\hline Salmas & 38.22 & 44.85 & 1337 & 2955 & Zabol & 31.03 & 61.48 & 489 & 883 \\
\hline Saman & 32.45 & 50.93 & 2057 & 2541 & Zahak & 30.90 & 61.68 & 495 & 838 \\
\hline Sanandaj & 35.33 & 47.00 & 1373 & 2244 & Zahedan & 29.47 & 60.88 & 1370 & 1093 \\
\hline Saqez & 36.25 & 46.27 & 1523 & 3015 & Zanjan & 36.68 & 48.48 & 1663 & 2884 \\
\hline Sarab & 37.93 & 47.53 & 1682 & 3517 & Zarand & 30.80 & 56.57 & 1670 & 1422 \\
\hline Sarableh & 33.78 & 46.57 & 1045 & 1513 & Zarineh $\mathrm{Ob}$ & 36.07 & 46.92 & 2143 & 3814 \\
\hline Sarakhs & 36.53 & 61.17 & 235 & 1516 & Zarqan & 29.78 & 52.72 & 1596 & 1574 \\
\hline Sararud & 34.33 & 47.30 & 1362 & 2118 & & & & & \\
\hline
\end{tabular}

Then spline method was applied to construct interpolated surface from above discrete set of results, to provide below Atlases (Fig.2. and Fig.3.). 


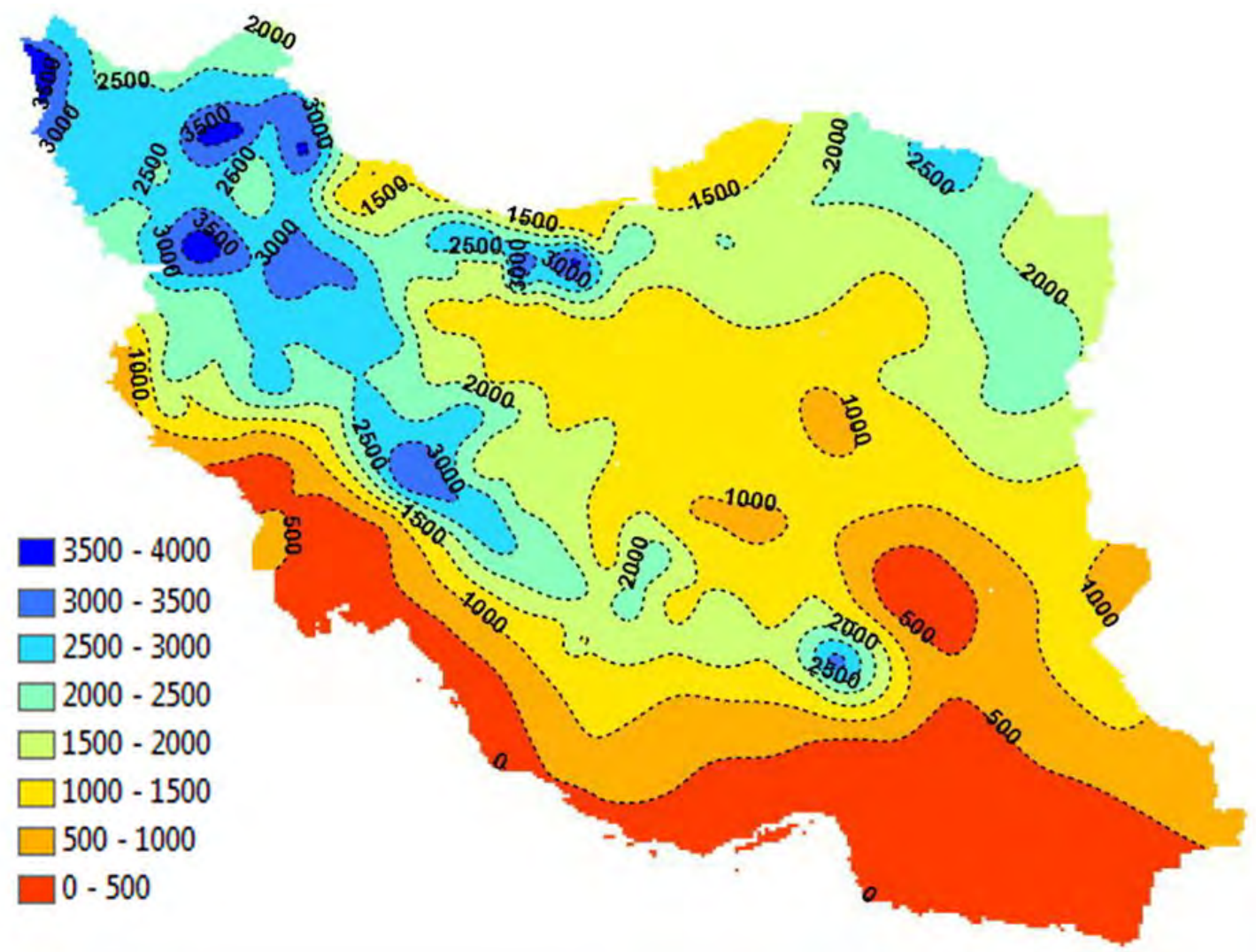

Fig. 2. Annual HDDs contours atlas over Iran entire zones, using spline interpolation

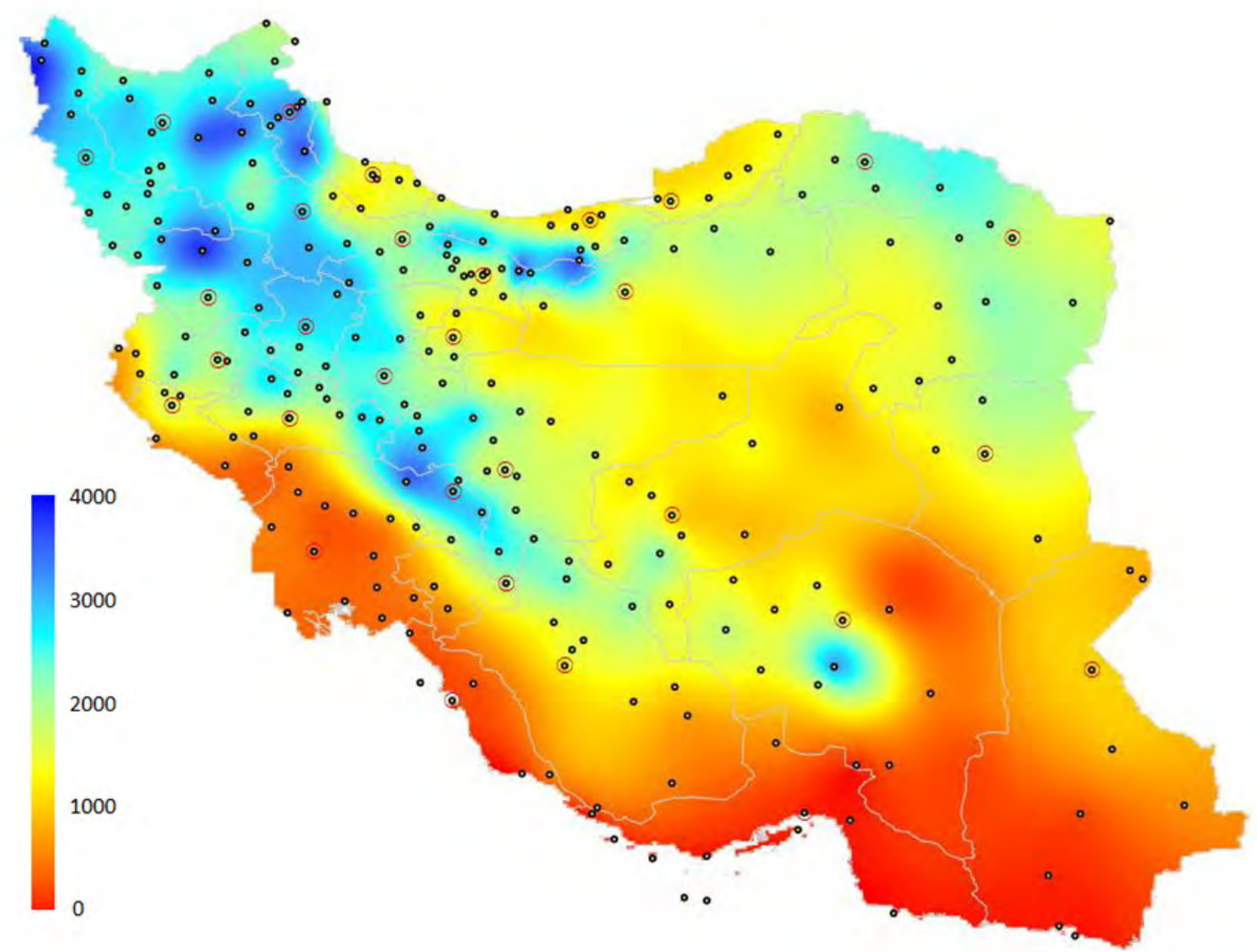

Fig. 3. Annual HDDs spectral atlas over Iran entire zones, using spline interpolation 
The highest value of HDDs is in Zarineh Obato mountainous city $\left(3814^{\circ} \mathrm{C}\right.$.day), in western region of Iran, and the lowest value of HDDs is in Abumusa island $\left(5^{\circ} \mathrm{C}\right.$.day), in the southern region. This large difference in HDDs shows the intense contrast in the climatic characteristics between these two extreme geographic points (The gheographical specifications of each point have been indicated in Table.1).

High values of HDDs can be observed in blue zones, mainly in northwestern regions of country, such as Chalderan $\left(3811^{\circ} \mathrm{C}\right.$.day), Khalkhal $\left(3566^{\circ} \mathrm{C}\right.$.day), and Ardebill $\left(3414^{\circ} \mathrm{C}\right.$.day), and some western regions such as Zarineh Obato $\left(3814{ }^{\circ} \mathrm{C}\right.$.day) as mentioned before. All of these points are located beside Zagros mountain range. In addition they also can be observed in northern regions such as Firuzkuh $\left(3479^{\circ} \mathrm{C}\right.$.day) because of locating beside Alborz mountain range.

Low values of HDDs can be observed in red zones, mainly in southern regions of country such as Jask Island $\left(10^{\circ} \mathrm{C}\right.$.day) and Chahbahar $\left(12^{\circ} \mathrm{C}\right.$.day $)$ because of Persian Gulf and Oman Sea climatic effects. They also can be observed in eastern regions of country such as Kahnuj $\left(304^{\circ} \mathrm{C}\right.$.day) because of locating beside Lut desert.

Fig.3 shows high contrast values of HDDs in Iran that introduces the exceptional climates over Iran. The uniqueness of these climates originates from several variables such as mountains and deserts especially Zagros and Alborz mountain ranges, Persian Gulf and Oman Sea, in addition unique deserts like Markazi and Lut. In country with these variations of climates, proposing appropriate HDDs can prevent higher escalation in energy consumption.

In Iran with governmental huge subsidies on $\mathrm{n}$ atural gas as a predominant heating energy carrier, government can set appropriate subsidies related on HDDs for each point of country.

\section{Conclusions}

HDDS over Iran entire zones, based on databases of 255 meteorological stations have been calculated and presented in a comprehensive table. Furthermore, contours and spectral atlas using spline interpolation have been demonstrated.

High contrast values of HDDs show the exceptional climates over Iran. In country with these variations of climates, proposing appropriate HDDs can prevent higher escalation in energy consumption.

\section{References}

[1] American Society of Heating, Refrigerating and Air-Conditioning Engineers, ASHRAE Handbook ,Fundamentals, Energy estimating and modeling methods, 2001, Ch.31 : Energy estimating and modeling methods.

[2] VDI, Economic efficiency of building installations, VDI 2067, Verein Deutscher Ingenieure.

[3] The chartered institution of building Services Engineers, Energy theory and applications, TM41-2006.

[4] Helmuth Späth, Two dimensional spline interpolation algorithms, A K Peters, Ltd., 1995. 